\title{
Coronary Heart Disease Concomitant with Atherosclerotic Cerebrovascular Disease
}

\author{
Yumei Liu, MD, PhD ${ }^{a, b}$, Beibei Liu, MD, MS ${ }^{a}$, Boyu Li, MD, PhD ${ }^{c}$, Yang Hua, MD ${ }^{a, b, *}$ \\ ${ }^{a}$ Department of Vascular Ultrasonography, Xuanwu Hospital, Capital Medical University, Beijing, China; ${ }^{b}$ Center of Vascular \\ Ultrasonography, Beijing Institute of Brain Disorders, Beijing, China; ${ }^{c}$ Department of Cardiology, Xuanwu Hospital, Capital Medical \\ University, Beijing, China \\ Received May 7, 2019; revision received July 10; accepted July 12.
}

\begin{abstract}
Cardio-cerebrovascular disease has a high rate of disability and mortality and affects a large number of people worldwide. Early prevention, accurate diagnosis and effective treatment are of great significance. This article reviews the risk factors, multimodalities of imaging diagnosis, and operational options for the atherosclerotic cardio-cerebrovascular combined disease.
\end{abstract}

Keywords: Cardio-cerebrovascular disease; Coronary heart disease; Cerebral ischemic stroke; Atherosclerosis

$\mathrm{R}$ eports on global, regional, and national disabilityadjusted life-years (DALYs) for 333 diseases and injuries and healthy life expectancy (HALE) for 195 countries and territories [1], demonstrated that ischemic heart disease and cerebrovascular disease are the main conditions affecting DALYs and HALE. Statistics from the American Heart Association (AHA) updated in 2018 [2] showed that coronary heart disease (CHD) and stroke contribute a lot to mortality, which causes significant economic burden all over the world. Recently, researchers in China reported that as many as 290 patients have cardiovascular disease (CVD), and stroke is the leading cause of death. Wang $\mathrm{Y}$ et al. [3] conducted a nationally representative door-to-door survey in 155 urban and rural centers in 31 provinces in China, and demonstrated that stroke in China has increased over the past 30 years, bringing heavy burden to families and societies. It is imperative to implement the prevention and treatment of cardio-cerebral vascular disease.

In 1947, Byer et al. [4] firstly reported that cerebral vascular disease could lead to cardiac damage and arrhythmia. After that, myocardial ischemia, arrhythmia, acute myocardial infarction or heart failure caused by acute stroke or brain trauma were identified as the cerebral-cardiac syndrome. Cerebral-cardiac syndrome increases the mortality of patients with cerebral vascular disease, and with the accelerating trend of population aging, morbidity and mortality have increased. Therefore, it is of great importance for patients to receive early risk assessment, accurate diagnosis and effective treatment of cerebrovascular disease, especially patients with CVD.

\section{Risks Factors of CHD Concomitant with}

\section{Cerebrovascular Disease}

The well-established risk factors of atherosclerosis are include age, sex, hypertension, diabetes mellitus (DM), hyperlipidemia (HLP) and smoking. Age-specific stroke rates are higher in men, but, because of longer life expectancy and much higher incidence at older ages, women have more stroke events than men [5]. HT is the most important independent risk factor for CVD death [2]. A prospective observational study found that every longterm decrease of 5-6 $\mathrm{mm} \mathrm{Hg}$ in diastolic blood pressure was associated with about $35-40 \%$ less stroke and $20-25 \%$ less CHD [6]. DM is an independent predictor for stroke recurrence. A meta-analysis showed that stroke recurrence

\footnotetext{
* Corresponding author: Department of Vascular Ultrasonography, Xuanwu hospital, the Capital Medical University, No. 45 Changchun Road, Xuanwu Hospital, Beijing 100053, China.

e-mail:dryanghua99@163.com unrestricted use, distribution and reproduction in any medium provided that the original work is properly attributed.
} 
risk of all stroke patients with DM was significantly higher than those without DM (HR, 1.45; 95\% CI, 1.32-1.59) [7]. HLP was found [8] increasing the risk of CHD and stroke that every $1 \mathrm{mmol} / \mathrm{L}$ increase in total cholesterol with $20 \%$ and $1 \%$ in women, $24 \%$ and $3 \%$ in men. Cigarette smoking also has a synergistic effect with other factors such as HLP, untreated HT and DM [2].

Studies showed that the prevalence of CHD concomitant with severe carotid artery stenosis (CAS) was $11.8 \%-29.8 \%$, increasing with the stenotic degree of CHD [9]. Meanwhile, the severity of CHD is positively correlated with the degree of carotid and intracranial artery stenosis [9]. Abd-Allah F et al [10] found that the prevalence of CHD concomitant with intracranial atherosclerosis was $11.9 \%$. In addition, multivessel or the left main coronary artery stenosis and moderate-tosevere extracranial CAS were the strongest predictors for the existence of intracranial atherosclerosis among CHD patients.

Beyond that, as an independent risk factor, CHD increases the risk of a stroke three times, and is a significant contributor to death among ischemic cerebral stroke (ICS) patients [10]. Taking patients without CAD as the reference, the age- and sex-adjusted hazard ratio of cardiac and cerebrovascular events was 4.36 (95\% CI, 1.35-14.12) for patients with asymptomatic CHD, and 6.86 (95\% CI, 2.15-21.91) for patients with known CHD after the incidence of ICS [11], which suggests the risk of recurrent CVD events will be high in patients with cardio-cerebral vascular combined disease.

Corresponding to the above, cardiogenic stroke is one of the major types of ICS, accounting for $30.2 \%$ [12]. The potential lesions included under this subtype are: mitral stenosis, prosthetic heart valve, myocardial infarction within the past four weeks, mural thrombus in the left cavities, left ventricular aneurysm, and atrial fibrillation. Compared with other subtypes of ICS, the highest recurrence rate was found due to cardioembolism (22\%; 95\% CI, 14.0-30.0), while the lowest 2 -year survival rate was in cardiogenic stroke $(55 \%$; 95\% CI, 0.47-0.63) [12]. As a consequence, early diagnosis and effective treatment are of particular importance among patients with cardio-cerebral vascular comorbidities.

\section{Imaging Examinations of CHD and Cerebrovascular Disease}

Cardio-cerebral artery disease can be comprehensively evaluated by multiple imaging modalities [13]. In recent years, ultrasonography, computed tomography angiography (CTA), magnetic resonance angiography (MRA) and digital subtraction angiography (DSA) have been widely used (Fig. 1).

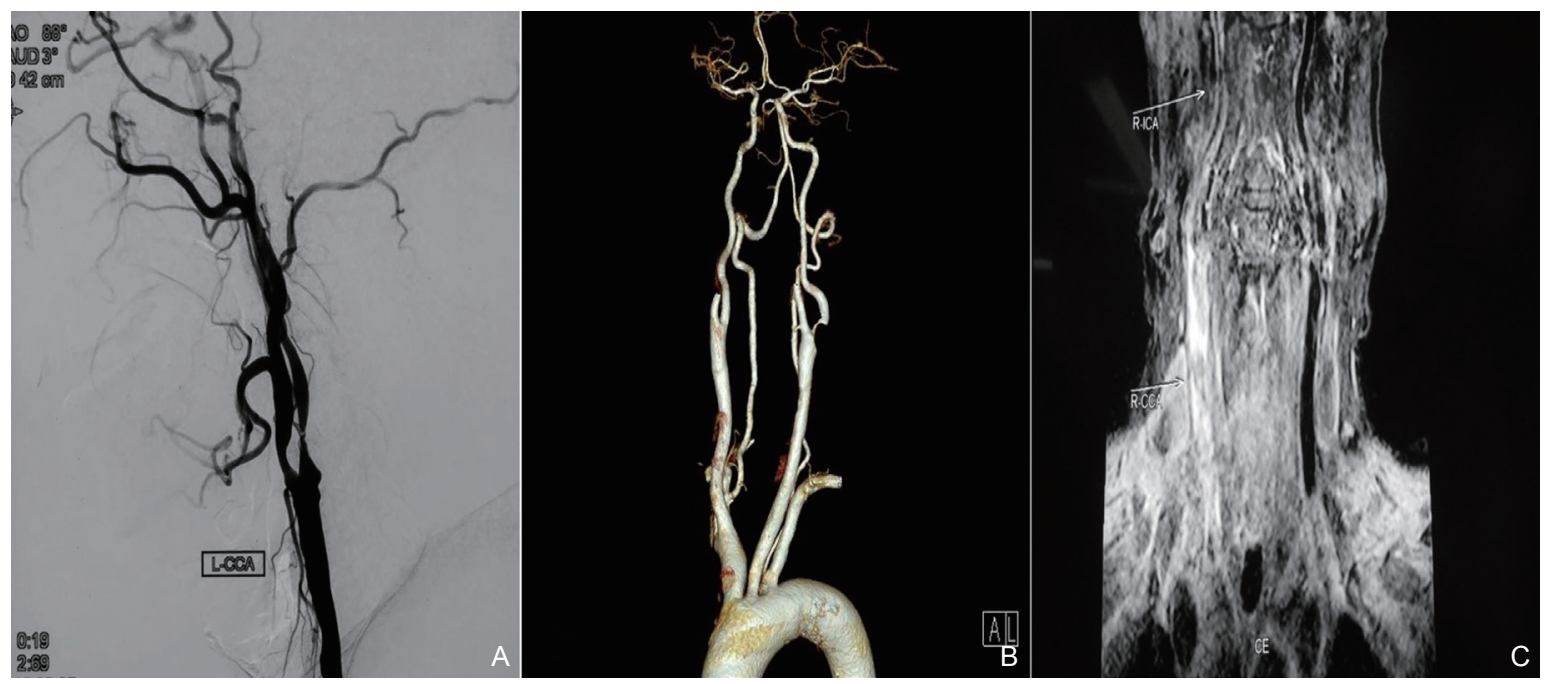

Figure 1 Different imaging modalities for cerebrovascular. (A) The severe stenosis was shown in the left internal carotid artery in DSA; (B) The severe stenosis was illustrated in the left internal carotid artery in CTA; (C) The near occlusion was shown in the right common carotid artery in high-resolution MRA.

\section{Imaging Examinations of CHD}

\section{CTA and DSA}

The severity of coronary artery lesions was defined as normal, mild stenosis (the stenotic degree of $<50 \%$ ), moderate stenosis $(50 \%-74 \%)$, severe stenosis $(\geqslant 75 \%$ for left anterior descending artery LAD, left circumflex artery LCX, right coronary artery RCA, or $\geqslant 50 \%$ for left main trunk), and occlusion [12].

Coronary CTA is widely used in clinical practice, with the sensitivity and specificity of CTA for coronary artery stenosis $84.9 \%$ and $97.2 \%$, respectively [14]. 
However, this imaging approach is susceptible to heart rate, abnormal heart rhythm or other factors. Also, caution must be used in patients with hypersensitivity to iodinated contrast media or renal insufficiency. Although coronary DSA is considered as the gold standard for CHD diagnosis, due to the high cost and invasive procedure, it is mainly used for revascularization of severe CHD (Fig. 2A, 2B).

\section{Intravascular Ultrasound (IVUS) and Optical Coherence Tomography (OCT)}

The development of IVUS [15] and OCT [16] revolutionized interventional cardiology. These techniques better visualized the vascular wall and played an important role in the diagnosis and treatment of CHD (Fig. 2C, 2D).

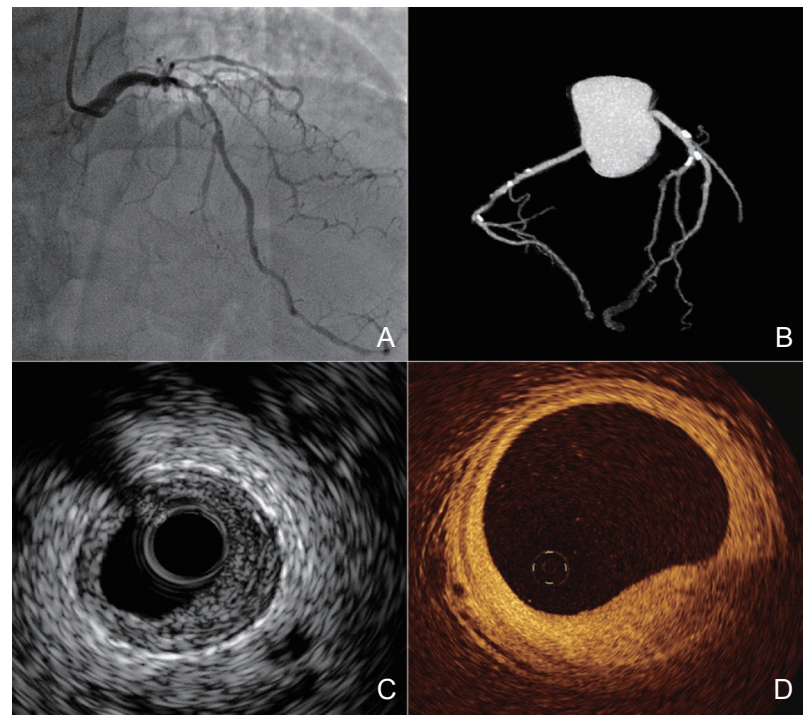

Figure 2 Coronary artery imaging. (A) The left main trunk with sever LAD stenosis was shown in DSA; (B) The left main trunk and the RCA with calcifications were illustrated by CTA; (C) The plaque was shown in a coronary artery by intravascular ultrasound; (D) OCT imaging for the intravascular plaque of coronary artery.

IVUS provides detailed morphology of intravascular atherosclerotic plaques including ulcers and the intraluminal thrombus, which help to identify high-risk plaques. According to this measurement, future major cardiovascular events can be predicted.

OCT is a technique using near-infrared light to provide microscopic insight into the vessels. According to the intensity of back-reflected near-infrared light, it measures the thickness of different biological tissues. Due to the high resolution $(10 \mu \mathrm{m})$, OCT is now considered as the gold standard for detecting vulnerable plaques. The application of OCT in cerebrovascular was reviewed [17] and was found to provide accurate diagnosis of atherosclerotic stenosis and to precisely measure the thin fibrous cap.
Imaging Examinations of Cerebrovascular Disease

\section{Ultrasonography and Contrast-enhanced Ultrasound (CEUS)}

Imaging examinations of extracranial and intracranial arteries include ultrasonography, CTA, MRA and DSA. Each modality has its advantages and disadvantages. When combined, they can provide comprehensive information on plaque and stenotic degree. Ultrasonography is the first line in the clinic for screening and monitoring carotid atherosclerosis due to its noninvasiveness, cost-effective, and convenience.

Primary B-mode ultrasound helps to identify carotid intima-media thickness (CIMT) and plaque, with color Doppler and spectral analysis, it can also grade the carotid stenosis (Fig. 3A, 3B, 3C). CIMT is an early manifestation of atherosclerosis. Salonen et al. [18] reported that for each $0.1 \mathrm{~mm}$ increase of CIMT, the risk of myocardial infarction increased by $11 \%$. Carotid plaque formed as the progression of atherosclerosis. Inaba $\mathrm{Y}$ et al. [19] showed that the ultrasound assessment of carotid plaque, compared with that of CIMT, had a higher diagnostic accuracy for the prediction of future CHD events (OR 1.35, 95\% CI 1.10-1.82). And hypoechoic, irregular plaques are associated with future CVD events (OR 2.97, 95\% CI 1.85-4.78) [20].

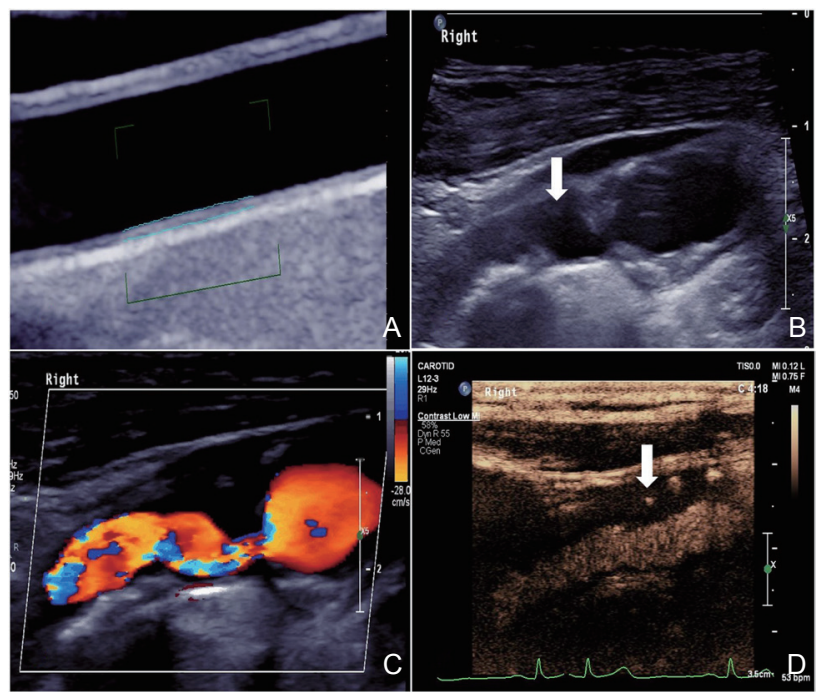

Figure 3 Ultrasonography exam for the carotid artery. (A) Intima-media thickness measurement; (B) By B-mode ultrasound, plaque ulceration was shown at the origin of the right carotid artery; (C) A severe stenosis due to atherosclerosis plaque was shown in the color mode ultrasound; (D) Neurovascular was detected by contrast-enhanced ultrasound.

Representing as a current new technique advance, CEUS can provide more advantages. It can accurately delineate the plaque, identify ulcerations, and differentiate total occlusion from the near occlusion. In 
addition, it also helps to detect and grade intraplaque neovascularization, explore vascular wall inflammation in patients with arteritis (Fig. 3D). Moreover, some specific ligands may attach to microbubbles that target molecules that achieve targeted therapy in the future [21].

\section{Transcranial Doppler (TCD) and Transcranial Color Doppler (TCCD)}

TCD or TCCD are generally used for detecting and quantifying intracranial artery stenosis. When combined, they may improve the diagnostic accuracy of intracranial artery stenosis [22]. A cross-sectional TCCD study on patients with advanced CAD showed that the asymptomatic intracranial stenosis rate was $63.6 \%$, and the most affected region was the $\mathrm{C} 1$ segment of the ICA [23]. TCCD was concluded to be a reliable method and to help to predict events in patients with advanced CAD.

Except for stenosis, micro-embolization is one of the important risks for stroke events that can be detected by TCD or TCCD. Potential sources of emboli include extracranial or intracranial plaques, which can usually be detected in the lesion side, while emboli from the aortic arch, cardiac chambers or coronary arteries can be detected in both sides of middle cerebral arteries. As we discussed above, cardio-embolism is the major reason for the recurrence of stroke. Therefore, TCD monitoring may help to stratify different risk for patients.

In brief, a comprehensive evaluation of the coronary artery and the extracranial and intracranial arteries has great clinical value for early diagnosis and effective treatment of cardio-cerebral vascular concomitant disorders.

\section{Therapeutic Strategies of CHD Concomitant with Cerebrovascular Disease}

In 1964, Garrett first performed coronary artery bypass grafting (CABG) successfully. Then in 1977, Grtientzig improved to perform percutaneous coronary intervention (PCI). Previous studies showed that patients with severe CAS undergoing CABG had an increased 9-fold higher mortality [24].

The optimal operative strategy in patients with severe CAS undergoing CABG is still unknown. Optional plans include carotid artery stenting or carotid endarterectomy (CEA), either simultaneously with PCI or CABG, or before/after PCI or CABG. The risk of any stroke or death in patients receiving synchronous CEA and CABG was 1.38-1.91 times higher than patients receiving isolated CABG [24]. However, Dick AM et al. [25] showed that synchronous CEA and CABG did not increase the risk of perioperative stroke relative to patients undergoing $\mathrm{CABG}$ alone. Illuminati $\mathrm{G}$ et al. [26] suggested that previous or simultaneous CEA in patients with unilateral severe asymptomatic CAS undergoing CABG could prevent stroke better than delayed CEA, without increasing the overall surgical risk.

For staging surgery, the interval time of $\mathrm{CABG}$ after carotid artery stenting is becoming the hotspot at present. In 2016, Dong et al. [27] suggested that the extent of CHD and the interval between carotid artery stenting and $\mathrm{CABG}$ were identified as independent risk factors for a major stroke, MI, or any death after the operation. An interval between carotid artery stenting and CABG of 6-30 days had the lowest risk of periprocedural cardiovascular events. Therefore, for patients with cardio-cerebral vascular disease, the individualized strategy should be made based on a comprehensive evaluation before.

\section{Conclusion}

In summary, early prevention, accurate diagnosis, systematic evaluation and effective treatment of CHD concomitant with CVD are of great significance in reducing the morbidity and improving long-term clinical efficacy.

\section{Funding}

This work was supported by Beijing Municipal Administration of Hospitals Clinical Medicine Development of Special Funding Support (ZYLX201706)

\section{Conflict of Interest}

The authors declare no conflict of interests.

\section{References}

[1] GBD 2016 DALYs and HALE Collaborators. Global, regional, and national disability-adjusted life-years (DALYs) for 333 diseases and injuries and healthy life expectancy (HALE) for 195 countries and territories, 1990-2016: a systematic analysis for the global burden of disease study 2016. Lancet 2017;390:1260-344.

[2] Benjamin EJ, Virani SS, Callaway CW, Chamberlain AM, Chang AR, Cheng S, et al. Heart disease and stroke statistics-2018 update: a report from the american heart association. Circulation 2018;137:e67-e492.

[3] Wang W, Jiang B, Sun H, Ru X, Sun D, Wang L, et al. Prevalence, incidence, and mortality of stroke in China: results from a nationwide population-based survey of 480687 adults. Circulation 2017;135:759-71.

[4] Byer E, Ashman R, Toth LA. Electrocardiograms with large, upright T waves and long Q-T intervals. Am Heart J 1947;33:796-806.

[5] Reeves MJ, Bushnell CD, Howard G, Gargano JW, Duncan PW, Lynch G, et al. Sex differences in stroke: epidemiology, clinical presentation, medical care, and outcomes. Lancet Neurol 2008;7:91526.

[6] Collins R, Peto R, MacMahon S, Hebert P, Fiebach NH, Eberlein $\mathrm{KA}$, et al. Blood pressure, stroke, and coronary heart disease. Part 2 , Short-term reductions in blood pressure: overview of randomised 
drug trials in their epidemiological context. Lancet 1990;335:827-38.

[7] Shou J, Zhou L, Zhu S, Zhang X. Diabetes is an Independent Risk Factor for Stroke Recurrence in Stroke Patients: A Meta-analysis. $J$ Stroke Cerebrovasc Dis 2015;24:1961-8.

[8] Peters SA, Singhateh Y, Mackay D, Huxley RR, Woodward M. Total cholesterol as a risk factor for coronary heart disease and stroke in women compared with men: A systematic review and meta-analysis. Atherosclerosis 2016;248:123-31.

[9] Hoshino T, Sissani L, Labreuche J, Ducrocq G, Lavallee PC, Meseguer E, et al. Prevalence of systemic atherosclerosis burdens and overlapping stroke etiologies and their associations with longterm vascular prognosis in stroke with intracranial atherosclerotic disease. JAMA Neurol 2018;75:203-11.

[10] Abd-Allah F, Kassem HH, Hashad A, Shamloul RM, Zaki A. Prevalence of intracranial atherosclerosis among patients with coronary artery disease: a 1-year hospital-based study. Eur Neurol 2014;71:326-30.

[11] Amarenco P, Lavallee PC, Labreuche J, Ducrocq G, Juliard JM, Feldman L, et al. Coronary artery disease and risk of major vascular events after cerebral infarction. Stroke 2013;44:1505-11.

[12] Kolominsky-Rabas PL, Weber M, Gefeller O, Neundoerfer B, Heuschmann PU. Epidemiology of ischemic stroke subtypes according to TOAST criteria: incidence, recurrence, and long-term survival in ischemic stroke subtypes: a population-based study. Stroke 2001;32:2735-40.

[13] Liu Y, Hua Y, Feng W, Ovbiagele B. Multimodality ultrasound imaging in stroke: current concepts and future focus. Expert Rev Cardiovasc Ther 2016;14:1325-33.

[14] Brodoefel H, Reimann A, Burgstahler C, Schumacher F, Herberts T, Tsiflikas I, et al. Noninvasive coronary angiography using 64-slice spiral computed tomography in an unselected patient collective: effect of heart rate, heart rate variability and coronary calcifications on image quality and diagnostic accuracy. Eur J Radiol 2008;66:134-41.

[15] Mintz GS, Nissen SE, Anderson WD, Bailey SR, Erbel R, Fitzgerald PJ, et al. American college of cardiology clinical expert consensus document on standards for acquisition, measurement and reporting of intravascular ultrasound studies (IVUS). a report of the american college of cardiology task force on clinical expert consensus documents. J Am Coll Cardiol 2001;37:1478-92.

[16] Terashima M, Kaneda H, Suzuki T. The role of optical coherence tomography in coronary intervention. Korean J Intern Med
2012;27:1-12.

[17] Chen CJ, Kumar JS, Chen SH, Ding D, Buell TJ, Sur S, et al. Optical coherence tomography: future applications in cerebrovascular imaging. Stroke 2018;49:1044-50.

[18] Salonen JT, Salonen R. Ultrasound B-mode imaging in observational studies of atherosclerotic progression. Circulation 1993;87:II56-65.

[19] Inaba Y, Chen JA, Bergmann SR. Carotid plaque, compared with carotid intima-media thickness, more accurately predicts coronary artery disease events: a meta-analysis. Atherosclerosis 2012;220:128-33.

[20] Jashari F, Ibrahimi P, Bajraktari G, Gronlund C, Wester P, Henein MY Carotid plaque echogenicity predicts cerebrovascular symptoms: a systematic review and meta-analysis. Eur J Neurol 2016;23:1241-7.

[21] Rafailidis V, Charitanti A, Tegos T, Destanis E, Chryssogonidis I. Contrast-enhanced ultrasound of the carotid system: a review of the current literature. J Ultrasound 2017;20:97-109.

[22] Yang J, Hua Y, Li X, Gao M, Li Q, Liu B, et al. The assessment of diagnostic accuracy for basilar artery stenosis by transcranial colorcoded sonography. Ultrasound Med Biol 2018 May;44:995-1002.

[23] Valaikiene J, Ryliskyte L, Valaika A, Puronaite R, Dementaviciene J, Vaitkevicius A, et al. A high prevalence of intracranial stenosis in patients with coronary artery disease and the diagnostic value of transcranial duplex sonography. J Stroke Cerebrovasc Dis 2019;28:1015-21

[24] Weimar C, Bilbilis K, Rekowski J, Holst T, Beyersdorf F, Breuer M, et al. Safety of simultaneous coronary artery bypass grafting and carotid endarterectomy versus isolated coronary artery bypass grafting: a randomized clinical trial. Stroke 2017;48:2769-75.

[25] Dick AM, Brothers T, Robison JG, Elliott BM, Kratz JM, Toole JM, et al. Combined carotid endarterectomy and coronary artery bypass grafting versus coronary artery bypass grafting alone: a retrospective review of outcomes at our institution. Vasc Endovascular Surg 2011;45:130-4

[26] Illuminati G, Ricco JB, Calio F, Pacile MA, Miraldi F, Frati G, et al. Short-term results of a randomized trial examining timing of carotid endarterectomy in patients with severe asymptomatic unilateral carotid stenosis undergoing coronary artery bypass grafting. $J$ Vasc Surg 2011;54:993-9.

[27] Dong H, Jiang X, Peng M, Zou Y, Che W, Qian H, et al. The interval between carotid artery stenting and open heart surgery is related to perioperative complications. Catheter Cardiovasc Interv 2016;87:564-9 